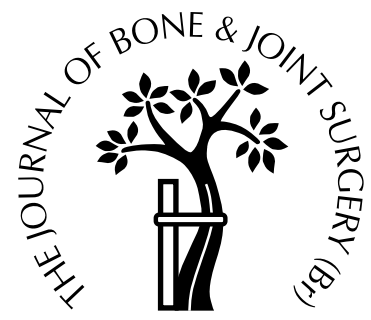

\title{
The regeneration of sensory neurones in the reconstruction of the anterior cruciate ligament
}

\author{
M. Ochi, J. Iwasa, Y. Uchio, N. Adachi, Y. Sumen \\ From Shimane Medical University, Izumo, Japan
}

W e examined whether somatosensory evoked potentials (SEPs) were detectable after direct electrical stimulation of injured, reconstructed and normal anterior cruciate ligaments (ACL) during arthroscopy under general anaesthesia. We investigated the position sense of the knee before and after reconstruction and the correlation between the SEP and instability.

We found detectable SEPs in all ligaments which had been reconstructed with autogenous semitendinosus and gracilis tendons over the past 18 months as well as in all cases of the normal group. The SEP was detectable in only 15 out of 32 cases in the injured group, although the voltages in the injured group were significantly lower than those of the controls. This was not the case in the reconstructed group. The postoperative position sense in 17 knees improved significantly, but there was no correlation between it and the voltage. The voltage of stable knees was significantly higher than that of the unstable joints. Our findings showed that sensory reinnervation occurred in the reconstructed human ACL and was closely related to the function of the knee.

J Bone Joint Surg [Br] 1999;81-B:902-6.

Received 15 June 1998; Accepted after revision 18 January 1999

The incidence of injury to the anterior cruciate ligament (ACL) during sport has increased. It is estimated that in the USA 95000 patients sustain such an injury each year of whom 50000 require reconstruction. ${ }^{1,2}$ About 2000 articles on the ACL have been published. ${ }^{3}$ It functions, along with other anatomical structures around the knee, to maintain static and dynamic equilibrium and it has an important role

M. Ochi, MD, Professor and Chairman

J. Iwasa, MD, Assistant Professor

Y. Uchio, MD, Assistant Professor

N. Adachi, MD, Assistant Professor

Y. Sumen, MD, Assistant Professor

Department of Orthopaedic Surgery, School of Medicine, Shimane Medi-

cal University, 89-1 Enyacho, Shimaneken 693-8501, Izumo, Japan.

Correspondence should be sent to Dr M. Ochi.

(C)1999 British Editorial Society of Bone and Joint Surgery 0301-620X/99/59202\$2.00 in proprioceptive feedback. Orthopaedic surgeons have focused mainly on how to reconstruct the ligament. Recently, it has been demonstrated histologically that there are several types of mechanoreceptor in the human ACL. ${ }^{4-8}$ Neural elements comprise approximately $1 \%$ of the area. ${ }^{9}$ These mechanoreceptors such as Pacinian corpuscles and Ruffini endings are thought to contribute to the position sense of the joint by feedback and reflex muscular activity. $^{10-15}$

Using an electrophysiological technique in nine patients, Pitman et al ${ }^{16}$ demonstrated that cortical somatosensory evoked potentials (SEPs) could be recorded after direct electrical stimulation of the normal ACL. These are elicited by electrical or mechanical stimulation to peripheral nerves and also allow assessment of the entire length of the somatosensory pathway from the peripheral nerve to the cerebral cortex including the posterior columns of the spinal cord. They concluded that their results provided direct evidence of the presence of a proprioceptive function in the intact ACL. Valeriani et $\mathrm{al}^{17}$ reported that seven of ten patients with a decreased position sense due to ACL insufficiency presented loss of the cortical P27 potential which reflects proprioceptive different inputs from the knee after stimulation of the common peroneal nerve above the articular nerve serving the ACL.

Our aim was to examine whether cortical SEPs are detectable after direct electrical stimulation of the injured or the reconstructed ACL. We compared the shape of the SEP with that from the normal ACL and examined the correlation between it and the instability and position sense of the injured and reconstructed knees.

\section{Patients and Methods}

Between January 1997 and March 1998, we studied 73 knees in 73 patients who had given informed consent to undergo intraoperative monitoring of the SEP. Four patients were excluded from analysis of the results because of technical problems. The remaining 69 patients were divided into three groups: the ACL-deficient group (32), the ACLreconstructed group (23) and the control group (14) (Table I). In the ACL-deficient group the ligament had been ruptured but the remnants were seen to bridge the femur and tibia although the sites of attachment were not anatom- 
Table I. Details of the patients in the three groups who had intraoperative monitoring of the SEP after electrical stimulation of the ACL and PCL

\begin{tabular}{lllll}
\hline & $\begin{array}{l}\text { Number of } \\
\text { patients }\end{array}$ & Male:female & Right:left & $\begin{array}{l}\text { Mean ( } \pm \text { SD) age } \\
\text { (yr; range) }\end{array}$ \\
\hline Deficient group & 32 & $16: 16$ & $19: 13$ & $25.5 \pm 9.3(15$ to 49) \\
Reconstructed group & 23 & $13: 10$ & $12: 11$ & $27.8 \pm 10.0(17$ to 47) \\
Control group & 14 & $9: 5$ & $4: 10$ & $22.9 \pm 12.3$ (11 to 58) \\
\hline
\end{tabular}

ical and the diameter of the tissue was small. The reconstruction group had received doubled semitendinosus and gracilis tendons in 22 knees and allogeneic fascia lata in one. They were examined at a minimum of 18 months after reconstruction. The control group with a normal ACL had had arthroscopy for internal derangement of the knee without abnormality in five knees, for meniscal injury in five and for a superficial chondral lesion in four.

Before arthroscopy, laxity was measured using the KT2000 knee arthrometer (MED Metric Corporation, San Diego, California) with a force of $133 \mathrm{~N}$ applied with the knee flexed at $30^{\circ}$. Both knees were tested and the laxity recorded as the difference in millimetres. The other knee was always normal. The position sense of the knee was tested to examine the ability of the patients to restore an angle at which the joint had been placed previously; this test was then modified according to the technique of Skinner et al. ${ }^{18}$ The examiner extended one of the patients' legs at a slow steady rate of approximately $10^{\circ}$ from a starting angle of $90^{\circ}$. The leg was stopped at a random angle of between $5^{\circ}$ and $25^{\circ}$ and held by the examiner for three seconds. The knee was then returned to the starting angle, and the examiner asked the subject to return the leg to the previous position. Inaccuracy was recorded as the difference between the perceived angle and the actual angle of flexion. Both knees were measured ten times and the mean inaccuracy was expressed as the error for the operated knee minus that recorded for the normal side.

Somatosensory evoked potentials. The patients were anaesthetised using nitrous oxide, oxygen and fentanyl citrate $(0.1 \mathrm{ml} / \mathrm{kg})$. Inhalation anaesthetic agents such as halothane, isofluorane and enflurene were not used because of their depressant effects on cortical responses. No local anaesthesia or epinephrine was used.

The ACL was electrically stimulated using a bipolar electrode probe with a Teflon-coated tip (AU-1, InterMedical Co Ltd, Tokyo, Japan) inserted into the midportion through an anteromedial portal. The stimulus comes only from the extreme tip of the probe and does not spread through the synovial fluid or physiological saline to other structures of the knee.

The stimulus used was a square wave of $0.2 \mathrm{~ms}$ duration at an intensity of $10 \mathrm{~mA}$ and rate of 3/s. Electrode impedence was maintained at between 2 and $10 \mathrm{k} \Omega$. Room temperature was maintained between 23 and $25^{\circ} \mathrm{C}$. The cortical response was recorded over a bandwidth of 0.5 to $1500 \mathrm{~Hz}$ for a duration of $100 \mathrm{~ms}$. Two hundred epochs were averaged; two responses were recorded for each trial and superimposed to ensure consistency with a measurement system for evoked potentials (Synax 1100; NEC Medical Systems, Tokyo, Japan).

The SEP was monitored by a surface electrode placed on the scalp at a point $2 \mathrm{~cm}$ lateral and $2 \mathrm{~cm}$ posterior to the $\mathrm{CZ}$ position according to the international 10 to 20 system with reference to the FP and ear. The ACL was electrically stimulated by a bipolar electrode probe. A peak or trough in each SEP (usually P1 trough or N1 peak) was chosen as the feature for programmed automatic detection for a monitoring session. In addition, SEPs produced by stimulating the posterior cruciate ligament (PCL) were recorded under the same conditions. The values of the latency and potential of P1 and N1 induced by PCL electrical stimulation were referred to as the control. The latency of P1 and N1 and the difference of potentials between $\mathrm{P} 1$ and $\mathrm{N} 1$ were calculated as the voltage of the SEP. The ratio of the voltage of the SEP induced by stimulation of the ACL to that induced by stimulation of the PCL was calculated. To determine the reproducibility of the SEPs the recording was repeated at least twice. The higher voltage was selected as being the most representative.

Statistical analysis. All numerical data were expressed as the mean \pm SD. Differences in parameters such as the latency and potential of $\mathrm{P} 1$ and $\mathrm{N} 1$, and the voltage ratio between the three groups were assessed using one-way analysis of variance. If a statistical difference existed, Scheffe's post-hoc test was used. The degree of correlation between the potential of the SEP, instability and position sense were estimated using Pearson's correlation coefficient (r). Student's unpaired $t$-test was used to compare the voltage between the stable knees and the unstable knees in the reconstructed group and the paired $t$-test to compare the position sense after operation with that before. A p value of less than 0.05 was regarded as statistically significant.

\section{Results}

SEP induced by electrical stimulation of the ACL. In the ACL-deficient group, reproducible cortical SEPs were detected only in 15 out of 32 knees and in the reconstruction group in 22 out of 23 knees (Fig. 1). The one patient in whom an SEP was not detected had an allograft 18 months previously. In the control group, reproducible SEPs were detected in all 14 knees.

Table II shows the data on the P1 and N1 latency and the voltage of the SEP obtained after stimulation of the ACL and PCL. The voltage of the SEP in the ACL-deficient 
Table II. SEP of the three groups after electrical stimulation of the ACL and PCL. The number in each group represents the patients with a positive SEP

\begin{tabular}{|c|c|c|c|c|c|c|c|}
\hline & \multicolumn{3}{|l|}{ ACL } & \multicolumn{3}{|l|}{ PCL } & \multirow{3}{*}{$\begin{array}{l}\text { Ratio of ACL voltage } \\
\text { to PCL voltage }\end{array}$} \\
\hline & \multicolumn{2}{|c|}{ Latency (ms) } & \multirow{2}{*}{$\begin{array}{l}\text { Voltage } \\
(\text { P1N1 }(\mu \mathrm{V}))\end{array}$} & \multicolumn{2}{|c|}{ Latency (ms) } & \multirow{2}{*}{$\begin{array}{l}\text { Voltage } \\
(\text { P1N1 }(\mu \mathrm{V}))\end{array}$} & \\
\hline & P1 & N1 & & P1 & N1 & & \\
\hline Defici & $30.5 \pm 8.3$ & $42.5 \pm 9.3$ & $0.74 \pm 0.34$ & $30.3 \pm 7.1$ & $39.5 \pm 8.5$ & $1.44 \pm 0.52$ & $0.61 \pm 0.48$ \\
\hline Reconstructed group $(n=22)$ & $30.8 \pm 7.9$ & $49.1 \pm 11.8$ & $1.14 \pm 0.45$ & $32.8 \pm 5.8$ & $43.2 \pm 8.0$ & $1.14 \pm 0.42$ & $1.08 \pm 0.40$ \\
\hline Control group $(n=14)$ & $29.9 \pm 7.8$ & $47.3 \pm 14.2$ & $1.51 \pm 0.73$ & $31.7 \pm 7.4$ & $42.0 \pm 9.0$ & $1.37 \pm 0.66$ & $1.30 \pm 0.86$ \\
\hline
\end{tabular}

Cortical SEP

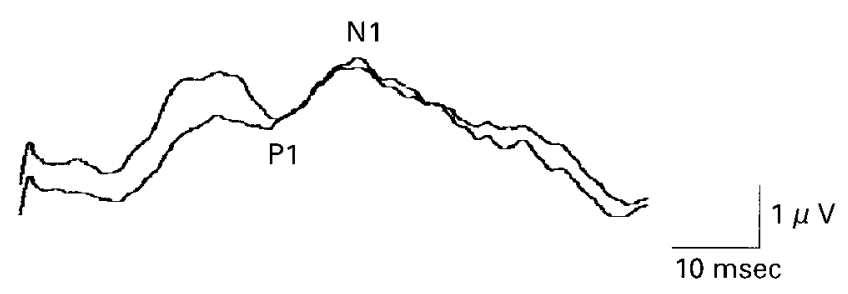

Fig. 1

Cortical SEP of a 20-year-old girl whose left ACL had been reconstructed 24 months before. Reproducible SEPs had been established with a trough (P1) and a peak (N1) in the initial component of the response. Although the voltages between $\mathrm{P} 1$ and $\mathrm{N} 1$ are $1.22 \mathrm{~V}$ and $1.19 \mathrm{~V}$. the higher voltage of $1.22 \mathrm{~V}$ is selected as the representative one.

group was significantly lower than that in the control group ( $p=0.001)$, but that in the ACL-reconstructed group was not $(p=0.128)$. There was no significant difference in the P1 or the N1 latency between any pairs in the three groups. Concerning the P1 and N1 latency and the voltage obtained after stimulation of the PCL there was no significant difference between pairs in any of the three groups.

Knee instability. The mean side-to-side difference of anterior displacement as measured by the KT-2000 knee arthrometer in the three groups was $5.33 \pm 2.04 \mathrm{~mm}, 2.18 \pm$ $2.27 \mathrm{~mm}$ and $0.89 \pm 2.25 \mathrm{~mm}$, respectively. The difference in the ACL-deficient group was significantly larger than

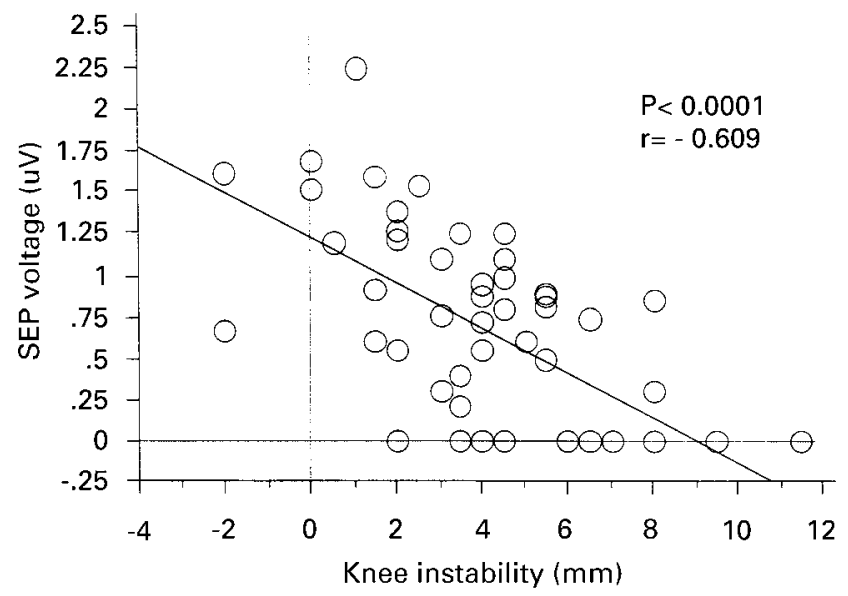

Fig. 2

Correlation between the voltage of the SEP and instability of the knee. There is a significant inverse correlation between the voltage and the sideto-side difference of anterior displacement in the deficient and reconstructed groups. that of the remaining two groups. There was no significant difference between the reconstructed and the control groups. There was a significant inverse correlation between the voltage of the SEP from the ACL and the side-to-side difference of anterior displacement in the deficient and reconstructed groups (Fig. 2). In the reconstructed group, the voltage of the SEP in the control knees with a side-toside difference of anterior displacement of less than $3 \mathrm{~mm}$ was significantly higher than that in the unstable knees ( 8 knees) with a side-to-side difference of $3 \mathrm{~mm}$ or more $(\mathrm{p}=0.035)$ (Fig. 3).

Joint position sense. The mean inaccuracy of the joint position sense was $1.01 \pm 1.24^{\circ}$ in the ACL-deficient group, $0.57 \pm 1.53^{\circ}$ in the reconstructed group, and $0.19 \pm 0.87^{\circ}$ in the control group. The inaccuracy of the ACL-deficient group was significantly higher than that of the other two groups. There was no significant difference in the values between the reconstructed and the control groups. In 17 knees in which the joint position sense was examined before and after operation, the preoperative inaccuracy was $2.15 \pm 1.45^{\circ}$ which improved significantly to $0.58 \pm 1.68^{\circ}$ $(\mathrm{p}<0.01)$ after operation. Fourteen of 17 knees showed an improvement in joint position sense after reconstruction. In both the deficient and the reconstructed groups the larger the inaccuracy of the joint position sense, the lower was the voltage from the ACL, but this was not statistically significant (Fig. 4).

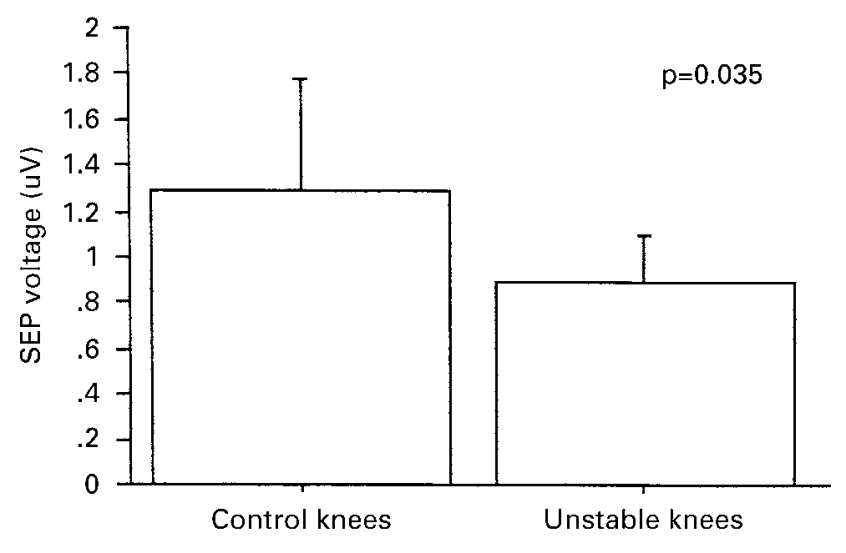

Fig. 3

Comparison of the voltage of the SEP between the stable and unstable groups after reconstruction. The side-to-side difference of anterior displacement after application of a force of $133 \mathrm{~N}$ was less than $3 \mathrm{~mm}$ in the control knee group and $3 \mathrm{~mm}$ or more in the group with unstable knees. 


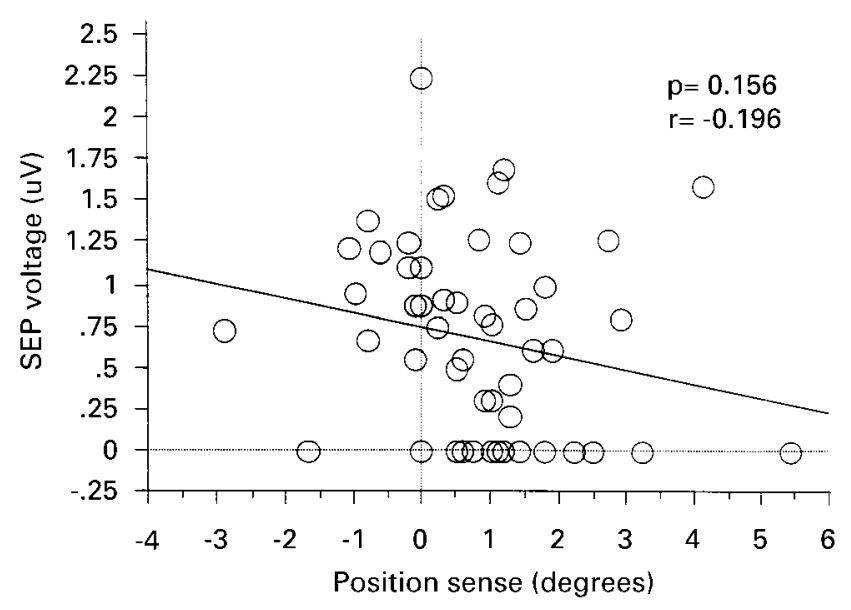

Fig. 4

Correlation between the voltage of the SEP and the position sense. There is a suggestion that the greater the inaccuracy of the joint position sense, the lower is the voltage in the deficient and reconstructed roups.

\section{Discussion}

Our study of the SEP has clearly shown that sensory neurones can regenerate in the reconstructed ACL and that the original sensory neurones are preserved in the ACL remnants to some extent. The voltage of the SEP of the reconstructed ACL was almost identical to that of the control group, although it was not shown that the voltage necessarily indicated the volume and the number of sensory neurones. The fact that electrical stimulation of the reconstructed ACL or the ACL remnant could induce SEPs indicates that sensory neurones in the ACL may provide information regarding deformation or deformity of the ligament, which may be a contributing factor to joint stability. The important function of ligaments is to stabilise the knee by way of the mechanoreceptor feedback system as well as to have a passive role as a mechanically strong restraint in preventing abnormal translation of the tibia. Thus, reconstruction of the ACL is not simply the reconstruction of a mechanically strong ligament but also of knee function.

The main focus of orthopaedic surgeons has been the reconstruction of a mechanically strong restraint to prevent abnormal translation, although in 1982 Kennedy, Alexander and Hayes ${ }^{19}$ pointed out that the clinical signs of ACL deficiency such as giving way, meniscal tear, progressive laxity, and early degenerative changes are due not only to a kinematic abnormality but also to loss of mechanoreceptor feedback. Studies on the functioning of grafted material after implantation in the knee have measured the maturation of the collagen fibrils of the biopsied tissue, the tension of the grafted tissue and stability using the KT-2000 knee arthrometer. $^{20,21}$

There are several reports concerning the presence of mechanoreceptors such as Ruffini endings, the Golgi tendon organ, and the Pacinian corpuscle in the ACL. ${ }^{22,23}$ The Ruffini and Golgi receptors are thought to be slow-adapting receptors which respond to a change in position rather than sensing acceleration or deceleration. A functional connection between ACL mechanoreceptors and the posterior articular and sciatic nerves has been demonstrated by Kraupse, Schmidt and Schaible. ${ }^{24}$ They showed that afferent nerve fibres from the ACL are activated by local pressure to the ligament or by certain movements of the knee, suggesting that mechanoreceptors provide information about tension of the ACL. Barrack et al ${ }^{10}$ examined the proprioceptive function of the knee in patients with injury to the ACL and concluded that poor results of proprioception were attributable to the loss of the ACL rather than other variables.

Our results clearly showed poor joint position sense and a low incidence of positive SEPs in the ACL-deficient group and, by contrast, an improvement of joint position sense and the positive potential in all but one knee after reconstruction, indicating that there may be a correlation between them.

Barrett ${ }^{11}$ examined proprioception in 45 patients after reconstruction of the ACL and showed that although their proprioceptive function was improved it was still inferior to that of normal patients. Co et $\mathrm{al}^{14}$ supported these findings. In dogs Barrack et $\mathrm{al}^{25}$ showed that the SEP returned in two out of six animals and that the free patellar graft contained neural elements six months after reconstruction of the ACL.

The position sense of the knee cannot be examined in animal studies but measurement of the SEP and the improvement in position sense are demonstrable in human studies. The return of sensory neurones in the ACL cannot be examined owing to lack of biopsied tissue. There have been no reports on the SEP after direct stimulation of the reconstructed ACL in clinical cases.

Our study showed that sensory reinnervation occurs in the reconstructed ACL and that voltage of the SEP inversely correlates with the degree of instability. Reconstruction of the ACL is not only reconstruction of a mechanical restraint but also of knee function.

No benefits in any form have been received or will be received from a commercial party related directly or indirectly to the subject of this article.

\section{References}

1. Miyasaka KC, Daniel DM, Stone ML, Hirshman P. The incidence of knee ligament injuries in the general population. Am J Knee Surg 1991:4:3-8.

2. Johnson DL, Fu FH. Anterior cruciate ligament reconstruction: why do failures occur? In: AAOS Instructional Course Lectures. Rosemont, Illinois: The American Academy of Orthopaedic Surgeons, 1995;44: 391-406.

3. Frank CB, Jackson DW. The science of reconstruction of the anterior cruciate ligament. J Bone Joint Surg [Am] 1997;79-A:1556-76.

4. Denti M, Monteleone M, Berardi A, Panni AS. Anterior cruciate ligament mechanoreceptors: histologic studies on lesions and reconstruction. Clin Orthop 1994;308:29-32.

5. Halata Z, Hans J. The ultrastucture of sensory nerve endings in anterior cruciate ligament. Anat Embryol 1989;179:415-21.

6. Krauspe R, Schmitz F, Zöller G, Drenckhahn D. Distribution of neurofilament-positive nerve fibres and sensory endings in the human anterior cruciate ligament. Arch Orthop Trauma Surg 1995;114:194-8. 
7. Schultz RA, Miller DC, Kerr CS, Micheli L. Mechanoreceptors in human cruciate ligaments: a histological study. J Bone Joint Surg [Am] 1984;66-A:1072-6.

8. Yahia LH, Newman N. Mechanoreceptors in the canine anterior cruciate ligament. Anat Anz 1991;173:233-8.

9. Schutte MJ, Dabezies EJ, Zimny ML, Happel LT. Neural anatomy of the human anterior cruciate ligament. J Bone Joint Surg [Am] 1987;69-A:243-7.

10. Barrack RL, Skinner HB, Buckley SL. Proprioception in the anterior cruciate deficient knee. Am J Sports Med 1989;17:1-6.

11. Barrett DS. Proprioception and function after anterior cruciate reconstruction. J Bone Joint Surg [Br] 1991;73-B:833-7.

12. Beard DJ, Kyberd PJ, Fergusson CM, Dodd CAF. Proprioception after rupture of the anterior cruciate ligament: an objective indication of the need for surgery? J Bone Joint Surg [Br] 1993;75-B:311-5.

13. Corrigan JP, Cashman WF, Brady MP. Proprioception in the cruciate deficient knee. J Bone Joint Surg [Br] 1992;74-B:247-50.

14. Co FJ, Skinner HB, Cannon WD. Effect of reconstruction of the anterior cruciate ligament on proprioception of the knee and the heel strike transient. J Orthop Res 1993;11:696-704.

15. Johansson H, Sjölander P, Sojka P. Receptors in the knee joint ligaments and their role in the biomechanics of the joint. Crit Rev Biomed Eng 1991;18:341-68.

16. Pitman MI, Nainzadeh $\mathbf{N}$, Menche $D$, et al. The intraoperative evaluation of the neurosensory function of the anterior cruciate ligament in humans using somatosensory evoked potentials. Arthroscopy 1992;8:442-7.
17. Valeriani M, Restuccia D, Lazzaro V Di, et al. Central nervous system modifications in patients with lesion of the anterior cruciate ligament of the knee. Brain 1996;119:1751-62.

18. Skinner HB, Barrack RL, Cook SD, Haddad RJ Jr. Joint position sense in total knee arthroplasty. J Orthop Res 1984;1:276-83.

19. Kennedy JC, Alexander IJ, Hayes KC. Nerve supply of the human knee and its functional importance. Am J Sports Med 1982;10: 329-35.

20. Daniel DM, Stone ML, Sachs R, Malcom L. Instrumented measurement of anterior knee laxity in patients with acute anterior cruciate ligament disruption. Am J Sports Med 1985;13:401-7.

21. Ochi M, Yamanaka T, Sumen Y, Ikuta Y. Arthroscopic and histologic evaluation of anterior cruciate ligaments reconstructed with the Leeds-Keio ligament. Arthroscopy 1993;9:387-93.

22. Freeman MA, Wyke B. The innvervation of the knee joint: an anatomical and histological study in the cat. J Anat 1967;101: 505-32.

23. Hogervorst T, Brand RA. Current concepts review. Mechanoreceptors in joint function. J Bone Joint Surg [Am] 1998;80-A: 1365-78.

24. Kraupse R, Schmidt M, Schaible H. Sensory innervation of the anterior cruciate ligament: an electrophysiological study of the response properties of single identified mechanoreceptors in the cat. $J$ Bone Joint Surg [Am] 1992;74-A:390-7.

25. Barrack RL, Lund PJ, Munn BG, Wink C, Happel L. Evidence of reinnervation of free patellar tendon autograft used for anterior cruciate ligament reconstruction. Am J Sports Med 1997;25:196-202. 